\title{
Characterisation of Linear and Nonlinear Noise of a Dual-Stage Broadband Discrete Raman Amplifier
}

\author{
M. A. Iqbal, L. Krzczanowicz, M. A. Z. Al-Khateeb, I. D. Philips, M. Tan, P. Harper and W. Forysiak \\ Aston Institute of Photonic Technologies (AIPT), Aston University, B4 7ET, Birmingham, UK \\ iqbalm7@aston.ac.uk
}

\begin{abstract}
The linear and nonlinear noise performance of a 70nm, dual-stage, 20dB-gain discrete Raman amplifier is characterised experimentally using different second-stage fibres. An advantageous IDF-SMF configuration is identified with an improvement in the nonlinear performance by $>1 d B Q^{2}$ compared with IDF-DCF configurations.
\end{abstract}

\section{Introduction}

In this paper, we report the experimental characterisation of the linear and nonlinear noise of a high output power and high gain broadband (1530-1600nm) dual-stage discrete Raman amplifier (DRA). We show that using inverse dispersion fibre (IDF) as the $1^{\text {st }}$ stage, and standard single mode fibre (SMF) rather than dispersion compensating fibre (DCF) as the $2^{\text {nd }}$ stage, gives a good balance between low noise figure (NF) and nonlinear noise induced transmission penalties.

In the design of DRAs, small core area fibres with higher Raman gain co-efficient (i.e. DCFs, highly nonlinear fibres (HNLFs), and IDFs) rather than conventional SMFs are usually used to improve the pump conversion efficiency. However, the noise performance of high gain DRAs is strongly impacted by double Rayleigh scattering (DRS) induced multi-path interference (MPI) crosstalk which also limits the maximum net gain up to $15 \sim 17 \mathrm{~dB}$ in single stage DRAs ${ }^{1}$.

Dual-stage DRAs can limit the generation of DRS using a mid-stage isolator and also provide higher net gain with low noise ${ }^{2}$. The choice of Raman gain fibre type and length in each stage is important to maintain improved noise and nonlinear performance ${ }^{3}$. Moreover, using gainfibres with high nonlinear index causes Kerr induced nonlinear penalties due to self-phase modulation (SPM) induced nonlinear phase shift (NPS), cross-phase modulation (XPM) and fourwave mixing (FWM), which can eventually degrade the performance of WDM polarisation- multiplexed (PM) phase modulated coherent transmission systems. So, careful design of high gain broadband DRAs with appropriate choice of Raman gain fibres is required for their optimum use in high capacity coherent WDM systems.

Here, we propose a dual-stage DRA design using $10 \mathrm{~km}$ of IDF as the $1^{\text {st }}$ stage, which was chosen for this purpose because it gives the optimal trade-off between NF, nonlinear impairments and gain efficiency compared with $\mathrm{DCF}$ and $\mathrm{SMF}^{3,4}$. We compare performance, in terms of NF and FWM product power, for the same net gain using $10 \mathrm{~km}$ of SMF, $5 \mathrm{~km}$ of DCF, and $10 \mathrm{~km}$ of DCF as the $2^{\text {nd }}$ stage. Finally, in a $5 \times 120 \mathrm{~Gb} / \mathrm{s}$ PM-QPSK WDM coherent transmission system consisting of a single $93.4 \mathrm{~km}$ span of SMF, we show our proposed IDF-SMF based DRA gives at least $1 \mathrm{~dB}$ improvement both in optimum launch power and $Q^{2}$ factor in the nonlinear regime compared with the DCF $2^{\text {nd }}$ stage based DRA schemes.

\section{Experimental setup}

Figure 1(a) shows the experimental setup of our backward pumped dual-stage DRA in which the $1 \mathrm{st}$ stage consists of $10 \mathrm{~km}$ IDF and the 2nd stage includes either $10 \mathrm{~km} \mathrm{SMF}$ or two different lengths $(5 \mathrm{~km}$ or $10 \mathrm{~km})$ of DCF. The $1 \mathrm{st}$ stage was backward pumped by five 1st order Raman pump laser diodes: 1425, 1444, 1462, 1476 and $1491 \mathrm{~nm}$ with $351,236,99,16$ and $142 \mathrm{~mW}$ pump power respectively to maintain $\sim 14 \mathrm{~dB}$ average net gain across $70 \mathrm{~nm}$. The $2 \mathrm{nd}$ stage was backward pumped with a similar pump set,

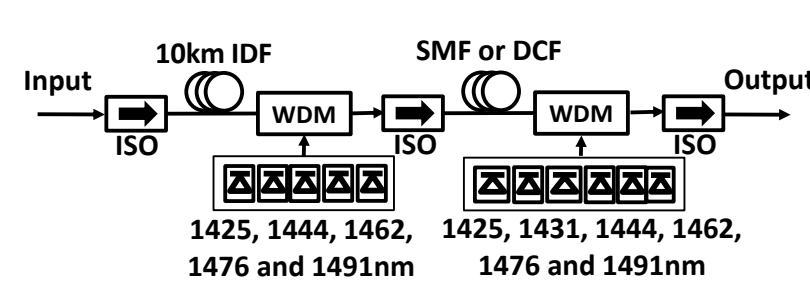

(a)

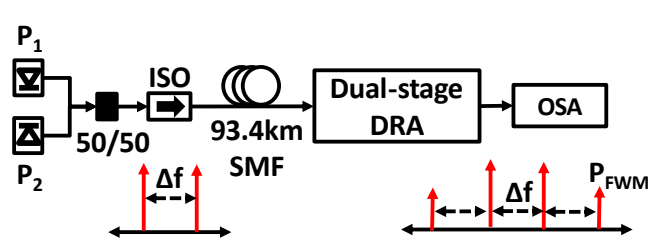

(b)

Fig. 1: (a) Schematic of dual-stage DRA schemes and (b) experimental setup of FWM product power measurement 
but with an additional $1431 \mathrm{~nm}$ pump to provide more pump power in the shortest pump wavelength region. The pump powers in the different $2^{\text {nd }}$ stages (Tab. 1) were adjusted to provide a total of $\sim 19.5 \mathrm{~dB}$ average net gain from the combined $1^{\text {st }}$ and $2^{\text {nd }}$ stages.

Tab. 1. Pump powers used in the 2nd stage

\begin{tabular}{|l|l|l|l|}
\hline $\begin{array}{l}\text { Pumps } \\
(\mathrm{nm})\end{array}$ & $\begin{array}{l}\text { SMF 10km } \\
(\mathrm{mW})\end{array}$ & $\begin{array}{l}\text { DCF 5km } \\
(\mathrm{mW})\end{array}$ & $\begin{array}{l}\text { DCF 10km } \\
(\mathrm{mW})\end{array}$ \\
\hline 1425 & 278 & 107 & 89 \\
\hline 1431 & 188 & 87 & 71 \\
\hline 1444 & 221 & 100 & 95 \\
\hline 1462 & 135 & 50 & 17 \\
\hline 1476 & 115 & 12 & 4 \\
\hline 1491 & 260 & 120 & 72 \\
\hline Total & 1197 & 476 & 348 \\
\hline
\end{tabular}

The net gain and NF over the $70 \mathrm{~nm}$ amplifier bandwidth were characterised using $24 \times 300 \mathrm{GHz}$ spaced channels of spectral shaped amplified spontaneous emission (ASE) from C and $L$ band EDFAs. Nonlinear performance of the different DRA schemes was characterised using a FWM efficiency measurement as shown in Fig. $1(b)^{5}$, where the $19.5 \mathrm{~dB}$ loss of the $93.4 \mathrm{~km}$ SMF transmission span was compensated using the DRA. Two tuneable $\mathrm{CW}$ lasers $\left(P_{1}\right.$ and $\left.P_{2}\right)$ at $3 \mathrm{dBm}$ power and a high resolution $(150 \mathrm{MHz})$ optical spectrum analyser (OSA) were used. The FWM efficiency was measured at the output of the DRA around 191.9THz, varying the frequency separation $(\Delta f)$ between the lasers from $1 \mathrm{GHz}$ to $50 \mathrm{GHz}$.

Finally, the performance of the three dualstage DRA schemes was characterised through a single span coherent WDM transmission experiment, using a standard $120 \mathrm{~Gb} / \mathrm{s}$ PMQPSK transmitter consisting of five $100 \mathrm{GHz}$ spaced WDM signals $(193.8 \sim 194.2 \mathrm{THz})^{6}$. At the transmitter, the WDM signals were amplified to $23 \mathrm{dBm}$ using a booster EDFA and the input signal power into the $93.4 \mathrm{~km}$ SMF span was varied from -5.7 to $12.3 \mathrm{dBm} / \mathrm{ch}$ using an input attenuator to maintain fixed input OSNR. The pump depletion in the $1^{\text {st }}$ stage was insignificant, so the same pump powers as used for NF characterisation were used in the $1^{\text {st }}$ stage. However, to ensure no drop-in signal power at the highest signal power levels due to pump depletion, the $2^{\text {nd }}$ stage multi-pump module was replaced by a high power $1455 \mathrm{~nm}$ fibre laser pump, and pump powers were adjusted at different signal powers to maintain a constant net gain of $\sim 19.5 \mathrm{~dB}$. A standard polarisation diverse coherent receiver with $80 \mathrm{GSa} / \mathrm{s}$ and $36 \mathrm{GHz}$ bandwidth was used at the receiver. The received signal was post-processed using offline digital signal processing (DSP) and $\mathrm{Q}^{2}$ factors were calculated from the error vector magnitude (EVM) of the received constellations.

\section{Results and discussion}

Firstly, the net gain and NF performance of the different DRA schemes were measured using the 24 spectrally shaped ASE channels over $70 \mathrm{~nm}$ at $-20 \mathrm{dBm} / \mathrm{ch}$ power. The average net gain and overall gain flatness were kept similar at $\sim 19.5 \mathrm{~dB}$ and $\sim 2.2 \mathrm{~dB}$ respectively for all the DRA schemes as shown in Fig. 2(a). The NF of all three schemes was found to be quite similar, as shown in Fig. 2(b), which is expected, given it is mainly dominated by the unchanged $1^{\text {st }}$ stage. The maximum NF of the IDF $10 \mathrm{~km} 1^{\text {st }}$ stage was $6.5 \mathrm{~dB}$ and $<1 \mathrm{~dB} \mathrm{NF}$ degradation was observed in the dual-stage DRAs. The NF tilt was due to the backward pumping configuration and could be further improved by using bidirectional pumping ${ }^{6,7}$. We also measured the net gain and output power keeping the pump power fixed in both stages and results are shown in Fig. 2(c). The saturated output power is $>22 \mathrm{dBm}$ in the IDF-SMF case, whereas the IDF-DCF cases showed saturation output powers reduced by at least $1.5 \mathrm{~dB}$, mainly due to more efficient power transfer from shorter to longer wavelength pumps and signals.

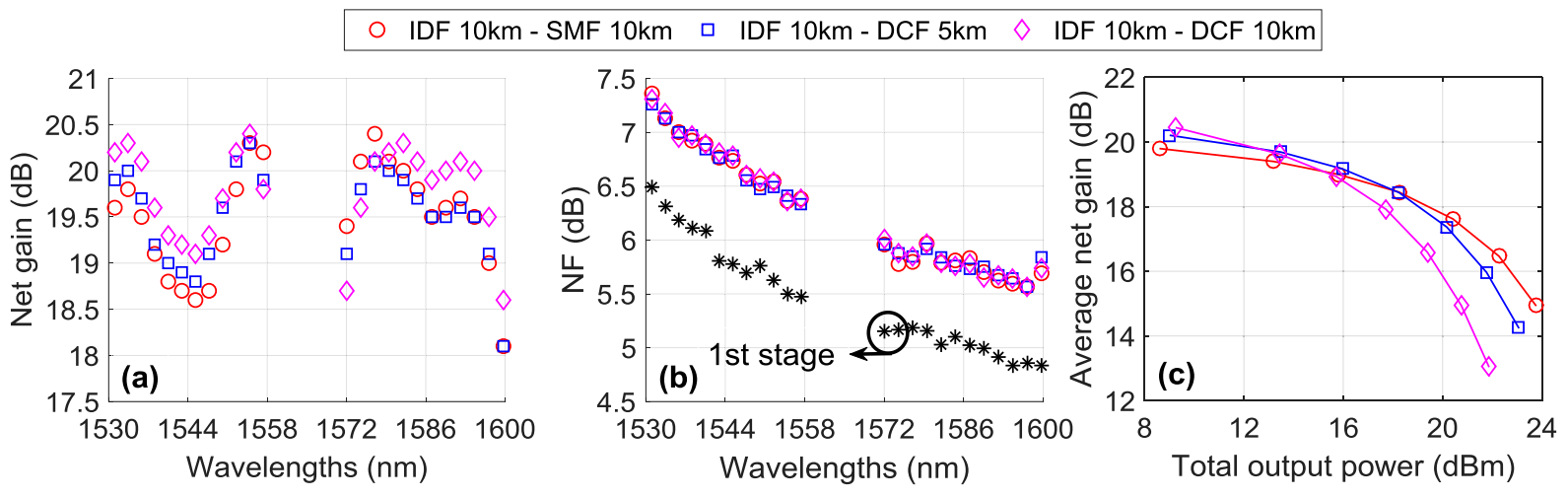

Fig. 2: Performance comparison among different DRA schemes: (a) net gain vs. wavelength; (b) NF vs. wavelength; and (c) average net gain over $70 \mathrm{~nm}$ bandwidth vs. total output power 


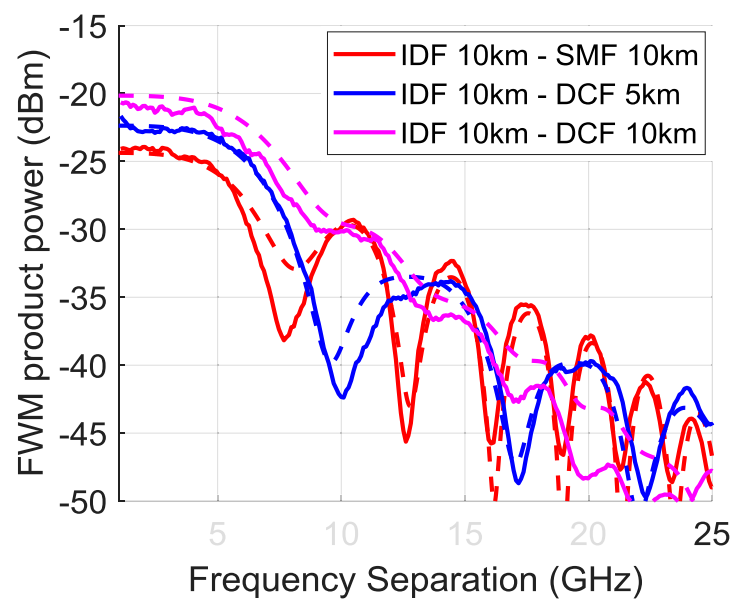

Fig. 3: Experimental (solid) and theoretical (dash) FWM product power as a function of the frequency separation between two $\mathrm{CW}$ lasers ( $3 \mathrm{dBm}$ each)

The nonlinear noise performance of the three DRA schemes were characterised both experimentally and theoretically ${ }^{5}$ in terms of FWM product power using the setup shown in Fig. 1(b). As expected, the results (Fig. 3) show that the DCF 2nd stage based DRAs gave higher FWM product power than that of SMF due to smaller core area and the resulting higher nonlinearity of the DCF. The IDF-SMF scheme showed $2.3 \mathrm{~dB}$ and $3.3 \mathrm{~dB}$ FWM product power reduction at the maximum values obtained at $1 \mathrm{GHz}$ frequency separation when compared with the IDF-DCF schemes with $5 \mathrm{~km}$ and $10 \mathrm{~km}$ of DCF, respectively. Similarly, the integrated FWM power over 1 to $15 \mathrm{GHz}$ gave $-18.8 \mathrm{dBm}$ which is $1.8 \mathrm{~dB}$ and $3.3 \mathrm{~dB}$ lower than that of the IDF-DCF $5 \mathrm{~km}$ and $10 \mathrm{~km}$ schemes, respectively.

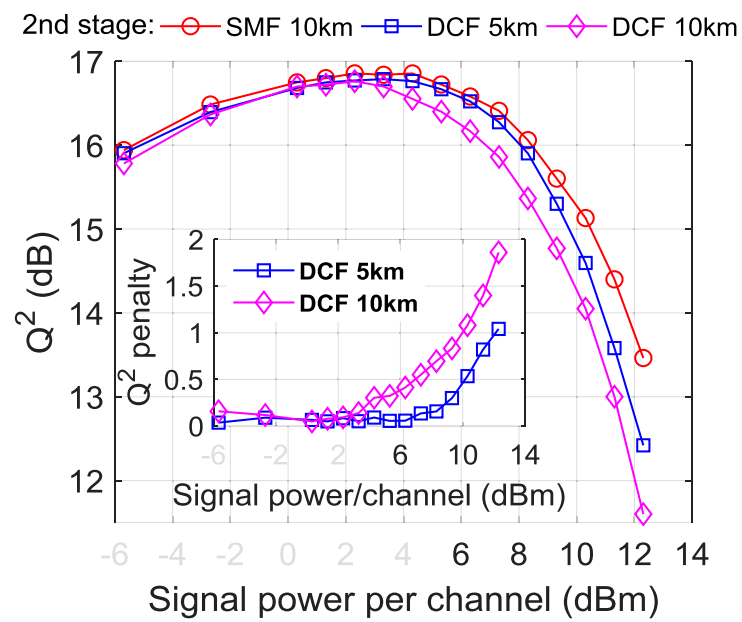

Fig. 4: $Q^{2}$ vs. signal power per channel for different dualstage DRA schemes (Insert: $Q^{2}$ penalty with respect to the best performed IDF $10 \mathrm{~km}$ - SMF $10 \mathrm{~km}$ based DRA)

Finally, the dual-stage DRA scheme was characterised using a single span coherent transmission experiment with $5 \times 120 \mathrm{~Gb} / \mathrm{s}$ PM-
QPSK signals. Fig. 4 shows the $\mathrm{Q}^{2}$ factor vs. signal launch power per channel for the centre WDM signal (194THz). Only five channels were chosen in order to maintain a sufficiently high launch signal power into the transmission span to observe and compare the nonlinear performance over a single span.

The IDF-SMF based DRA showed the maximum $\mathrm{Q}^{2}$ factor of $16.9 \mathrm{~dB}$ with $0.1 \sim 0.2 \mathrm{~dB}$ improvement compared with the DCF $2^{\text {nd }}$ stage based DRA schemes. In the amplifier noise limited linear regime, all the schemes showed similar performance which can be expected from the NF features shown in Fig. 2(b). However, significant $Q^{2}$ factor degradation was observed in the nonlinear regime at high signal powers due to the Kerr nonlinearities for the DCF $2^{\text {nd }}$ stage based schemes, as also supported by the FWM product power growth shown in Fig. 3 . The $Q^{2}$ factor penalties of the IDF-DCF DRAs compared with IDF-SMF based DRA are shown in the inset of Fig. 4, with maximum $Q^{2}$ factor degradation due to nonlinear penalties up to $1.9 \mathrm{~dB}$ and $1.1 \mathrm{~dB}$ for the IDF-DCF DRAs with $10 \mathrm{~km}$ and $5 \mathrm{~km}$ of DCF, respectively. In repeated systems, we expect these Kerr-induced nonlinearities will accumulate and DCF based DRAs will show much stronger $Q^{2}$ factor degradation than the SMF based DRA.

\section{Conclusions}

We have characterised a novel design of dualstage DRA in terms of linear and nonlinear noise performance. We have confirmed that while the linear noise (NF) is mainly dominated by the $1^{\text {st }}$ stage, the nonlinear noise induced penalties can be reduced significantly by using a larger core area fibre in the high power $2^{\text {nd }}$ stage. Our IDFSMF based DRA shows $2 \mathrm{~dB}$ optimum launch power improvement and up to $1.9 \mathrm{~dB} \mathrm{Q}^{2}$ factor improvement in the nonlinear regime compared with an IDF-DCF $10 \mathrm{~km} 2^{\text {nd }}$ stage based DRA.

\section{Acknowledgements}

The work is funded by EPSRC grants EP/M009092/1 and EP/L000091/1 and supported by II-VI Inc.

\section{References}

[1] D. Hamoir et al., OAA 2000, vol. 44, p. 61-63, OMD8.

[2] S. A. E. Lewis et al., IEEE Photon. Technol. Lett., vol. 12 , no. 5 , p. $528-530$ (2000).

[3] M. A. Iqbal et al., ICTON 2017, We.D5.4.

[4] L. Krzczanowicz et al., Opt. Express, vol. 26, p. 70917097 (2018).

[5] M. A. Z. Al-Khateeb et al., IEEE Photonics Conference (IPC) 2016, p. 795-796.

[6] M. A. Iqbal et al., Opt. Express, vol. 25, p. 27533-27542 (2017).

[7] S. Kado et al., ECOC 2001, PD.F.1.8. 